\title{
Direct versus indirect bypasses for adult ischemic-type moyamoya disease: a propensity score-matched analysis
}

\author{
*Xiaofeng Deng, MD,,-4 Faliang Gao, MD,-4 Dong Zhang, MD, ${ }^{1-4}$ Yan Zhang, MD,1-4 \\ Rong Wang, MD, ${ }^{1-4}$ Shuo Wang, MD, ${ }^{1-4}$ Yong Cao, MD, ${ }^{1-4}$ Yuanli Zhao, MD, ${ }^{1-4}$ Yuesong Pan, MD, ${ }^{2-5}$ \\ Xingju Liu, MD,1-4 Qian Zhang, MD, ${ }^{1-4}$ and Jizong Zhao, MD ${ }^{1-4}$

\begin{abstract}
${ }^{1}$ Department of Neurosurgery, Beijing Tiantan Hospital, Capital Medical University; ${ }^{2}$ China National Clinical Research Center for Neurological Diseases (NCRC-ND); ${ }^{3}$ Center of Stroke, Beijing Institute for Brain Disorders; ${ }^{4}$ Beijing Key Laboratory of Translational Medicine for Cerebrovascular Disease; and ${ }^{5}$ Department of Neurology, Beijing Tiantan Hospital, Capital Medical
\end{abstract} \\ University, Beijing, China
}

OBJECTIVE The optimal surgical modality for moyamoya disease (MMD) remains unclear. The aim of this study was to compare the surgical effects of direct bypass (DB) and indirect bypass (IB) in the treatment of adult ischemic-type MMD. METHODS Adult patients with ischemic-type MMD who underwent either DB or IB from 2009 to 2015 were identified retrospectively from a prospective database. Patients lost to follow-up or with a follow-up period less than 12 months were excluded. Recurrent stroke events and modified Rankin Scale (mRS) scores at the last follow-up were compared between the 2 surgical groups after 1:1 propensity score matching.

RESULTS A total of 220 patients were considered, including 143 patients who underwent DB and 77 patients who underwent IB. After propensity score matching, 70 pairs were obtained. The median follow-up period was 40.5 months (range 14-75 months) in the DB group and 31.5 months (range 14-71 months) in the IB group ( $p=0.004)$. Kaplan-Meier analysis showed that patients who received DB had a longer stroke-free time (mean 72.1 months) compared with patients who received IB (mean 61.0 months) $(p=0.045)$. Good neurological status (mRS score $\leq 2)$ was achieved in 64 patients in the DB group (91.4\%) and 66 patients in the IB group (94.3\%), but there was no significant difference $(p=$ 0.512).

CONCLUSIONS Although neurological function outcome was not determined by the surgical modality, DB is more effective in preventing recurrent ischemic strokes than IB for adult ischemic-type MMD.

https://thejns.org/doi/abs/10.3171/2017.2.JNS162405

KEY WORDS moyamoya disease; revascularization; direct bypass; indirect bypass; stroke; vascular disorders

$\mathrm{M}$ OYAMOYA disease (MMD) is a rare, chronic, and progressive occlusive cerebrovascular disorder of unknown etiology, which is characterized by stenosis or occlusion at the end of the bilateral internal carotid arteries and the initial portion of the middle and anterior cerebral arteries. As a compensation for internal carotid artery occlusion, small arterial collaterals (moyamoya vessels) form at the base of the brain, which appear as a "puff of smoke" on angiographic studies. 3,9,17,21,27,29 The disease is predominantly found in Asian populations. ${ }^{18}$
MMD can lead to ischemic or hemorrhagic strokes such as transient ischemia attack (TIA), cerebral infarction, and intraventricular hemorrhage. Depending on the manifestation, MMD is usually classified into 2 main phenotypes: ischemic or hemorrhagic. ${ }^{14,32}$

The ideal treatment of MMD should be effective in both improving symptoms and preventing recurrent strokes. In recent decades, bypass surgical procedures have been developed and are the most popular treatment strategy nowadays. The most commonly used surgical modalities

ABBREVIATIONS CB = combined bypass; $\mathrm{DB}=$ direct bypass; $\mathrm{DSA}=$ digital subtraction angiography; $\mathrm{IB}=$ indirect bypass; $\mathrm{MMD}=$ moyamoya disease; $\mathrm{mRS}=$ modified Rankin Scale; PCA = posterior cerebral artery; $Q A L Y=$ quality-adjusted life year; $T I A=$ transient ischemic attack.

SUBMITTED September 17, 2016. ACCEPTED February 9, 2017.

INCLUDE WHEN CITING Published online August 11, 2017; DOI: 10.3171/2017.2.JNS162405.

* Drs. Deng and Gao contributed equally to this work. 
include indirect bypass (IB), direct bypass (DB), and combined bypass (CB). However, the superior surgical modality remains a topic of debate, especially regarding the most classic procedures, DB and IB. In our opinion, the main shortcomings of previous studies are the small numbers of patients and the heterogeneity of the cohorts (for example, children or adults, ischemic-type or hemorrhagic-type MMD, and different angiographic findings), which might affect the accuracy of their conclusions.

Therefore, we conducted this study to analyze differences in the surgical effects between DB and IB in treating adult ischemic-type MMD, and we enrolled a large series of patients. To reduce the effects of heterogeneity, propensity score matching was performed and the clinical outcomes were compared between the matched surgical groups.

\section{Methods \\ Patient Selection}

The study protocol was approved by the Institutional Review Board of Beijing Tiantan Hospital, Capital Medical University, Beijing, China. All MMD patients (including patients with unilateral MMD) who received revascularization procedures at our institution from June 2009 to May 2015 were screened. Written informed consent was obtained from all patients. The diagnosis was based on the 2012 guidelines for MMD. ${ }^{11}$ Patients with moyamoya syndrome secondary to the identified etiologies were ruled out. Pediatric patients, adult hemorrhagic-type patients, patients who underwent $\mathrm{CB}$ or different bilateral surgical modalities (for example, DB on a single hemisphere and IB on the other hemisphere), and patients without preoperative digital subtraction angiography (DSA) studies were excluded (Fig. 1).

\section{Retrospective Chart Review}

Clinical data were retrospectively reviewed, including sex, age, hypertension, diabetes mellitus, smoking history, and clinical manifestations. Neurological status was evaluated with the modified Rankin Scale (mRS) on admission and at follow-up examination. ${ }^{21}$ Imaging characteristics included cerebral infarction on MRI, Suzuki stage, unilateral involvement of MMD, and posterior cerebral artery (PCA) involvement on DSA.

\section{Surgical Modalities}

Two kinds of surgical modalities were analyzed in this study: DB and IB. DB indicates the end-to-side anastomosis of the branches of the superficial temporal artery to the cortical branches of the middle cerebral artery $\left(\mathrm{M}_{4}\right.$ segment). IB included encephaloduroarteriosynangiosis and multiple bur holes. If both DB and IB were performed in 1 hemisphere, it was considered $\mathrm{CB}$ and these patients were excluded from this study. The operation time and surgical complications were noted.

\section{Follow-Up}

After discharge from the hospital, follow-up was conducted in the outpatient department or by telephone inter-

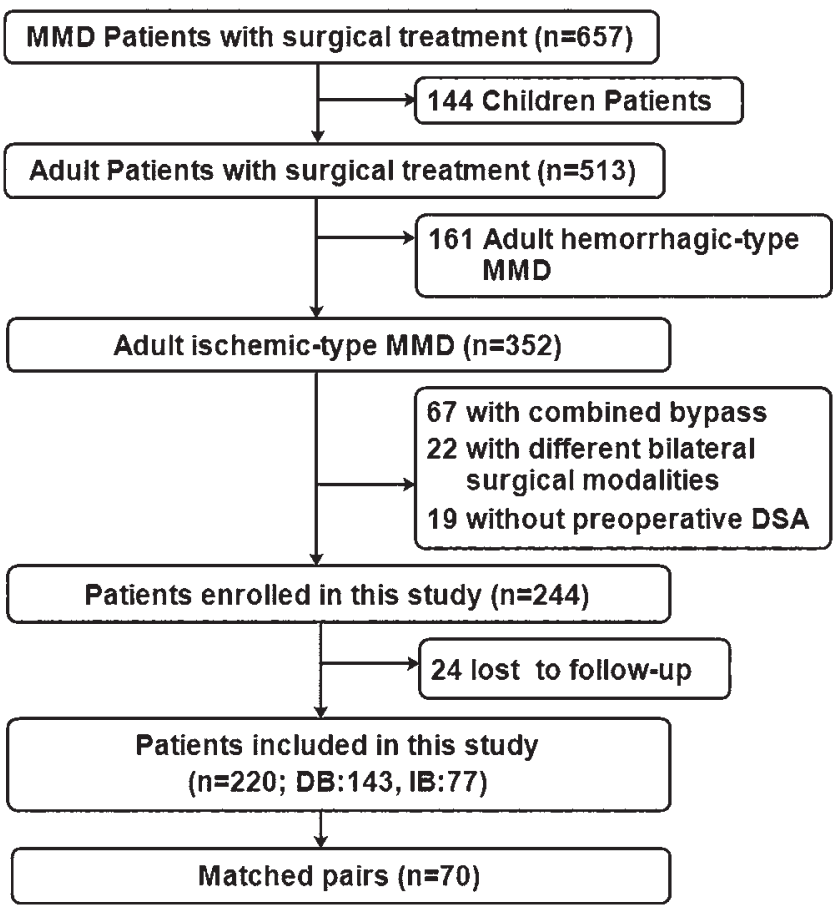

FIG. 1. Flow diagram showing selection of the study participants.

view. Patients who were lost to follow-up or those with a follow-up period less than 12 months were not included in this study. The primary outcome of interest was recurrent stroke, including TIA, cerebral infarction, and intracranial hemorrhage. mRS scores were also recorded to evaluate each patient's neurological status, which was classified as good (mRS score $\leq 2)$ or poor (mRS score $\geq 3$ ).

\section{Statistical Analysis}

All statistical analyses were performed with SPSS for Windows (version 22.0; IBM), with the R software (R Project for Statistical Computing, R-2.15.1-win.exe) and the plug-in (SPSS_Statistics_REssentials_22.0-FP1_win64. exe) that links with the corresponding version of SPSS, and the installed propensity score-matching package (PSMATCHING3.04.exe) (IBM). In this study, 1:1 propensity score matching was adopted to overcome the bias arising from the lack of randomization and the heterogeneity of the MMD patients. ${ }^{1,24}$ A multivariable logistic regression model was built to predict the probability of each patient being treated with DB or IB on the basis of the covariables, including age (grouped according to quartile), sex, hypertension, diabetes mellitus, smoking history, mRS score on admission, cerebral infarction, Suzuki stage, unilateral involvement of MMD, and PCA involvement. The distributions of the aforementioned characteristics in the 2 surgical groups were assessed with the standardized mean difference, which was calculated as the difference in the means or proportions of a variable divided by the pooled estimate of the standard deviation of that variable. ${ }^{26,30} \mathrm{Ac}-$ cordingly, a standardized mean difference of less than 0.1 indicates a negligible difference; a value between 0.1 and 0.3 indicates a small difference; a value between 0.3 and 
TABLE 1. Patient characteristics and group comparisons before and after propensity score matching

\begin{tabular}{|c|c|c|c|c|c|c|c|}
\hline \multirow[b]{2}{*}{ Characteristic } & \multicolumn{3}{|c|}{ Before Propensity Score Matching } & \multicolumn{4}{|c|}{ After Propensity Score Matching } \\
\hline & DB & IB & $p$ Value & DB & IB & $p$ Value & Standardized Mean Difference* \\
\hline No. of patients & 143 & 77 & & 70 & 70 & & \\
\hline \multicolumn{8}{|l|}{ Age, yrs $†$} \\
\hline $17-29$ & 26 & 27 & \multirow{4}{*}{0.006} & 14 & 23 & \multirow{4}{*}{0.068} & \multirow{4}{*}{0.095} \\
\hline $30-36$ & 33 & 17 & & 15 & 16 & & \\
\hline $37-44$ & 43 & 19 & & 23 & 18 & & \\
\hline $45-63$ & 41 & 14 & & 18 & 13 & & \\
\hline Male/female sex ratio & $77: 66$ & $27: 50$ & 0.008 & $32: 38$ & $26: 44$ & 0.303 & 0.171 \\
\hline Hypertension & $46(32.2)$ & $19(24.7)$ & 0.245 & $13(18.6)$ & $18(25.7)$ & 0.309 & 0.152 \\
\hline Diabetes & $13(9.1)$ & $6(7.8)$ & 0.744 & $5(7.7)$ & $5(7.7)$ & 1.000 & 0.000 \\
\hline Smoking & $11(7.7)$ & $7(9.1)$ & 0.319 & $4(6.1)$ & $6(9.4)$ & 0.428 & 0.031 \\
\hline \multicolumn{3}{|c|}{ mRS score on admission } & \multirow{6}{*}{0.100} & & & \multirow{6}{*}{0.740} & \multirow{6}{*}{0.000} \\
\hline 0 & $3(2.1)$ & $3(3.9)$ & & $1(1.4)$ & $3(4.3)$ & & \\
\hline 1 & $94(65.7)$ & $39(50.6)$ & & $44(62.9)$ & $37(52.9)$ & & \\
\hline 2 & $35(24.5)$ & $27(35.1)$ & & $16(22.9)$ & $24(34.3)$ & & \\
\hline 3 & $8(5.6)$ & $5(6.5)$ & & $7(10.0)$ & $4(5.7)$ & & \\
\hline 4 & $3(2.1)$ & $3(3.9)$ & & $2(2.8)$ & $2(2.8)$ & & \\
\hline Cerebral infarction & $101(70.6)$ & $51(66.2)$ & 0.501 & $52(74.3)$ & $47(67.1)$ & 0.353 & 0.156 \\
\hline \multicolumn{3}{|l|}{ Suzuki stage } & \multirow{6}{*}{0.024} & & & \multirow{6}{*}{0.808} & \multirow{6}{*}{0.057} \\
\hline II & $17(11.5)$ & $0(0.0)$ & & $6(8.6)$ & $0(0.0)$ & & \\
\hline III & $51(33.8)$ & $28(29.9)$ & & $22(31.4)$ & $28(40.0)$ & & \\
\hline IV & $47(33.8)$ & $27(20.8)$ & & $24(34.3)$ & $25(35.7)$ & & \\
\hline V & $23(16.9)$ & $20(23.4)$ & & $15(21.4)$ & $15(21.4)$ & & \\
\hline $\mathrm{VI}$ & $5(3.8)$ & $2(26.0)$ & & $3(4.3)$ & $2(2.9)$ & & \\
\hline Unilateral MMD & $23(16.1)$ & $6(7.8)$ & 0.138 & $5(7.1)$ & $6(8.6)$ & 0.753 & 0.034 \\
\hline PCA involvement & $35(24.5)$ & $26(33.8)$ & 0.142 & $22(31.4)$ & $21(30)$ & 0.855 & 0.033 \\
\hline
\end{tabular}

0.5 indicates a moderate difference; and a value above 0.5 indicates a considerable difference. ${ }^{26,30}$

Continuous data that met and did not meet the normal distribution were compared using the t-test and rank-sum test, respectively. Categorical variables were compared with the chi-square test. Ordinal variables (mRS scores and Suzuki stages) were analyzed with the rank-sum test. The stroke-free survival analysis was performed using Kaplan-Meier curves with log-rank statistics. A probability value of $<0.05$ was considered statistically significant.

\section{Results}

A total of 657 MMD patients who underwent revascularization procedures at Beijing Tiantan Hospital between 2009 and 2015 were identified, including 144 pediatric patients and 513 adult patients (Fig. 1). Among the 513 adult patients, we excluded 161 hemorrhagic-type patients, 67 ischemic-type patients who underwent $\mathrm{CB}, 22$ ischemictype patients who underwent different bilateral surgical modalities, 19 ischemic-type patients without preoperative DSA, and 24 patients who were lost to follow-up or who were followed up for less than 12 months. Finally, 220 adult patients with ischemic-type MMD were included in this study, including 143 patients who received DB and 77 patients who received IB.

\section{Baseline Characteristics Before and After Propensity Score Matching}

Patient characteristics before matching are shown in Table 1. Patients were significantly older in the DB group (range 17-63 years; median 38 years) than the IB group (range 17-56 years; median 34 years) $(\mathrm{p}=0.032)$. Patient ages were grouped by quartile before matching, and there was also a significant difference between the 2 groups ( $\mathrm{p}$ $=0.006$ ). The male/female ratio was significantly higher in the DB group (1:0.86) than the IB group (1:1.85) $(\mathrm{p}=$ $0.008)$. A significant difference was also detected in Suzuki stage $(\mathrm{p}=0.024)$. No significant differences were detected in the other characteristics, including hypertension, diabetes, smoking history, mRS score on admission, cerebral infarction, unilateral MMD, and PCA involvement $(\mathrm{p}>0.05)$. 
After propensity score matching, there were 70 patients in each group with equivalent demographic, clinical, and angiographic features. The $\mathrm{p}$ value of the overall balance test was 0.927 , and the relative multivariate imbalance L1 value was 0.882 before matching and 0.843 after matching, indicating good balance between the 2 surgical groups.

The details of the baseline characteristics of the surgical groups are described in Table 1. The median patient age was in the 40s in both groups. There was a female predominance, with a male/female ratio of 1:1.19 in the DB group and 1:1.69 in the IB group. According to the $\mathrm{mRS}$ grading system, the majority of patients had good neurological status on admission (mRS score $\leq 2$ ): $87.2 \%$ of patients in the DB group and $91.5 \%$ in the IB group. Most patients had cerebral infarction on preoperative MRI, including cortical infarction, subcortical infarction, and both cortical and subcortical infarction: $74.3 \%$ of patients in the DB group and $67.1 \%$ in the IB group. On preoperative DSA, most patients had Suzuki Stage III and IV MMD, which accounted for $65.7 \%$ and $75.7 \%$ of the patients in the DB group and IB group, respectively. After propensity score matching, there was no significant difference in age, sex, hypertension, diabetes, smoking history, mRS score on admission, cerebral infarction, Suzuki stage, unilateral MMD, and PCA involvement ( $p>0.05)$. Moreover, the results of the standardized mean difference also demonstrated negligible or small differences in all characteristics between the 2 groups (Table 1).

\section{Surgical Duration and Complications}

The surgical duration of the DB group (range 90-340 minutes; median 210 minutes) was significantly longer than that of the IB group (range 60-314 minutes; median 160 minutes) ( $p<0.001)$. Surgical complications were observed in 11 patients in the DB group (15.7\%), including new-onset cerebral infarction in 3 patients, intracranial hemorrhage in 1 patient, hyperperfusion syndrome (confirmed by the patient's symptoms and CT perfusion) in 2 patients, wound infection in 2 patients, and seizure in 3 patients. Surgical complications were observed in 9 patients in the IB group (12.9\%), including new-onset cerebral infarction in 3 patients, intracranial hemorrhage in 1 patient, subdural effusion in 2 patients, and seizure in 3 patients. No significant difference in the surgical complications was observed between the 2 groups $(\mathrm{p}=0.629)$.

\section{Clinical Outcome}

After propensity score matching, the DB group was observed for 14 to 75 months (median 40.5 months) and the IB group was observed for 14 to 71 months (median 31.5 months), and a significant difference was detected between the 2 groups $(\mathrm{p}<0.001)$. During the follow-up period, recurrent stroke events were observed in 9 patients in the IB group (12.9\%; 5 patients with TIA, 3 patients with cerebral infarction, and 1 patient with both TIA and cerebral infarction), which occurred at 10 to 57 months after surgery (median 17 months), and in 3 patients in the DB group (4.3\%; 1 patient with TIA and 2 patients with cerebral infarction), which occurred at 1 to 19 months after surgery (median 8 months). Kaplan-Meier analysis showed

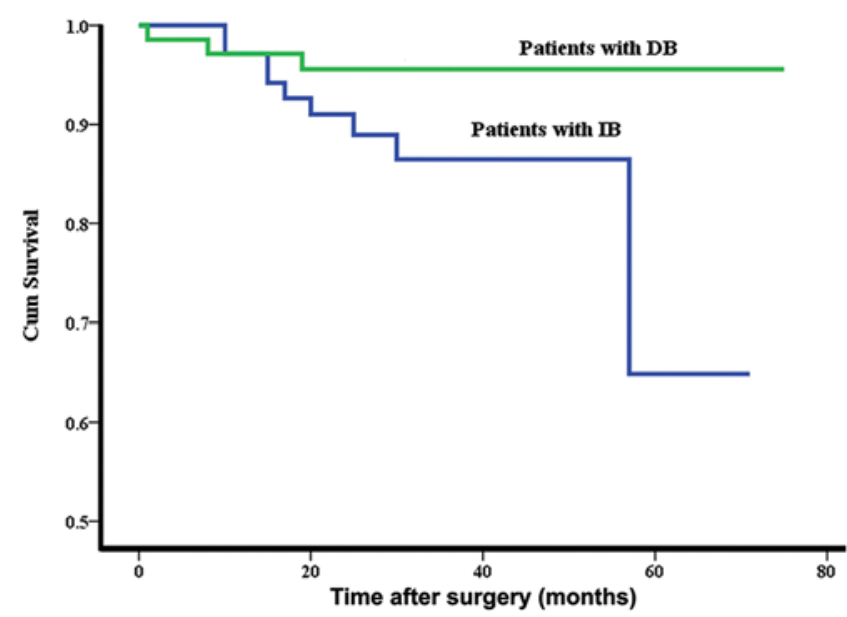

FIG. 2. Kaplan-Meier curve for stroke-free survival in the patients who underwent the 2 surgical modalities. Cum = Cumulative. Figure is available in color online only.

that the stroke-free time was significantly longer in the DB group (mean 72.1 months) than the IB group (mean 61.0 months) $(\mathrm{p}=0.045)$ (Fig. 2). At the last follow-up, good neurological status (mRS score $\leq 2)$ was observed in 64 patients in the DB group (91.4\%) and 66 patients in the IB group (94.3\%), but there was no significant difference between groups $(\mathrm{p}=0.512)$.

\section{Discussion}

MMD is a chronic and progressive occlusive cerebrovascular disorder than can occur in children and adults. There are a variety of manifestations such as TIA, cerebral infarction, intracranial hemorrhage, and seizure that can lead to disability or even death. Until now, there was no effective treatment for this disease. ${ }^{5,19}$ Revascularization surgery is the most popular treatment of MMD. However, its efficacy remains controversial because some studies have shown that bypass surgery does not even improve prognosis. ${ }^{12,25,31}$ Furthermore, there is no consensus about the detailed surgical procedure, and the optimal surgical modality is still uncertain, even when discussing the classic surgical modalities of DB and IB..$^{2,13,15,16,20-23,25}$ This is especially controversial in adult patients, as both $\mathrm{DB}$ and IB have shown good results in pediatric patients. We think different conclusions are reached by different studies because of the following reasons: the small numbers of included patients, the heterogeneity of the cohorts (for example, pediatric and adult patients, ischemic-type and hemorrhagictype MMD), and the lack of a randomized controlled study. Therefore, in this study, we focused on a special population (patients with adult ischemic-type MMD), enrolled a large series of patients, and used propensity score matching to reduce the effects of heterogeneity and reduce the effects of nonrandomization. Common factors that might have affected surgical effects were considered, including age, sex, hypertension, diabetes, smoking, mRS score on admission, cerebral infarction, Suzuki stage, unilateral MMD, and PCA involvement. The effects of the 2 most common surgical modalities, DB and IB, were compared. 
First, although the surgical duration of the DB group (range 90-340 minutes; median 210 minutes) was significantly longer than that in the IB group (range 60-314 minutes; median 160 minutes) $(\mathrm{p}<0.001)$, there was no significant difference in the surgical complications between the DB group (15.7\%) and the IB group (12.9\%) ( $\mathrm{p}=0.629)$. According to previous studies, both DB and IB have their advantages and disadvantages. The main advantage of DB is that it can directly improve cerebral hemodynamics immediately after surgery, with good revascularization on DSA images, especially in adult patients. $3^{3,4,9,20,22}$ However, the major disadvantage of $\mathrm{DB}$ is that it is a more challenging technique and requires good donor and recipient arteries of sufficient caliber. Additionally, surgical complications might be more common in patients undergoing DB because DB always requires a longer surgical duration and carries the risk of hyperperfusion syndrome, which could result in numbness of the limbs, motor weakness, or aphasia. ${ }^{6,8,9,13,17,20,22}$ In our study, although hyperperfusion syndrome was noted, its incidence was relatively low (2.9\%), and the DB group did not have a significantly higher incidence of surgical complications. This result was in accordance with previous studies, and a meta-analysis of 16 studies also demonstrated that there was no significant difference in perioperative complications. ${ }^{28}$

Second, recurrent stroke events were observed in 12 of all 140 patients, with a pooled incidence of $8.6 \%$. This is similar to other studies where the incidence of recurrent stroke events ranged from 5\% to $31 \% .^{6,7,10,20,22,31}$ Even if the follow-up period was shorter in the IB group (median 31.5 months) compared with the DB group (median 40.5 months), the incidence of recurrent stroke events was higher in the IB group (12.9\%) than the DB group (4.3\%). However, the Kaplan-Meier analysis also demonstrated a significantly longer stroke-free time in the DB group (mean 72.1 months) compared with the IB group (mean 61.0 months) $(\mathrm{p}=0.045)$. This was consistent with the study by Lee et al., which also showed that DB was more effective at preventing recurrent strokes than IB for patients with adult ischemic-type MMD $(\mathrm{p}<0.05){ }^{20}$ Moreover, in a meta-analysis published by Qian et al., ${ }^{28}$ DB had a greater efficacy for secondary stroke risk reduction compared with IB in ischemic-type MMD patients $(\mathrm{p}=0.01)$. However, some studies have come to different conclusions. For example, Macyszyn et al. recently performed a meta-analysis that included 33 studies and 4197 surgically treated hemispheres. ${ }^{22}$ This study enrolled 6 adult series, and 762 cases underwent DB and 1524 cases underwent IB. They drew the opposite conclusion of Lee et al., with IB being superior to DB. In our opinion, these contradictory conclusions might result from the heterogeneity of the disease and the different evaluation indicators. In the study by Macyszyn et al., the qualityadjusted life years (QALYs) were significantly higher in the IB group compared with the DB group. However, the expected QALYs in adults at the 4-year follow-up were 3.502 QALYs for the DB group and 3.553 QALYs for the IB group; thus, a difference of a 0.05 QALY was obtained. As Teo et al. discussed, although this difference might be statistically significant, whether this translates into clinical significance is doubtful. ${ }^{33}$ Moreover, the long-term risk of ischemic stroke in the IB group (10.5\%) far exceeded that in the DB group (1.4\%). This is consistent with our results. Therefore, we believe DB is still the better bypass procedure for preventing recurrent stroke events in adult patients.

Third, according to the mRS scores, the DB and IB groups had no significant difference in neurological outcome $(p=0.512)$. Good neurological status could be expected in both the DB group (91.4\%) and IB group (94.3\%). We think the different results between recurrent stroke events and neurological status might be attributed to the different mRS scores on admission and the large proportion of TIA patients in the IB group. First, although there was no significant difference in $\mathrm{mRS}$ scores on admission, the proportion of patients with poor neurological status was higher in the DB group (12.8\%) than the IB group (8.5\%). Second, although the incidence of recurrent strokes was higher in the IB group, the main recurrent stroke was TIA (5 of 9 patients in the IB group), which only resulted in transient symptoms and did not affect neurological outcome at the last follow-up. Therefore, we think the above-mentioned factors might have affected the statistical results, and we believe the 2 outcome indicators (neurological outcome and recurrent stroke events) need to be evaluated comprehensively.

This study has some limitations. First, although we used propensity score matching to reduce the effects of heterogeneity and reduce the effects of nonrandomization, this is not a randomized controlled trial. Some potential bias might still exist. For example, there is selection bias in choosing the surgical modality, which is usually due to the lack of a good recipient artery. Besides, although common factors that might have affected the clinical outcome were considered and the heterogeneity of these factors was reduced to a great extent by propensity score matching, there are also some factors that are not easily catalogued (such as the diameter and quality of the donor and recipient arteries, hemodynamic factors, extent of cerebral ischemia, etc.) and these might have affected the results. A randomized controlled trial comparing DB and IB for treating MMD would be the best method for determining the superior revascularization technique, but it is difficult to perform. This is also the reason we chose propensity score matching to analyze the results, which can reduce the bias arising from the lack of randomization to a great extent. Second, the follow-up period might not be long enough to draw a more positive conclusion. The $\mathrm{p}$ value was barely significant $(0.045)$ in the comparison of recurrent stroke events between the 2 groups. If a longer follow-up period was used, our results might have been more confirmative. Third, this study evaluated the 2 most common surgical procedures, DB and IB. However, CB is also performed in clinical practice, especially in recent years, and some authors have reported that CB has its advantages. ${ }^{6,34}$ This was not analyzed in the current study, and we will estimate its advantages and disadvantages in our future work.

\section{Conclusions}

For adult patients with ischemic-type MMD, DB is 
more effective at preventing recurrent ischemic stroke than IB, although neurological function outcome was not determined by the surgical modality.

\section{Acknowledgments}

We thank Drs. Jia Wang, Meng Zhao, Ziwen Yang, Peicong Ge, Chang Liu, Yonggang Ma, Cunxin Tan, Pengjun Jiang, Rutao Luo, Gongyi Yu, Zhiyong Shi, Lebao Yu, and Junshi Shao for their contributions to data collection. This study was supported by the "11th Five-Year Plan" and the "13th Five-Year Plan" National Science and Technology supporting plan (2006BAI01A13, 2015BAI12B04), the National Key Technology Research and Development Program of the Ministry of Science and Technology of China (2013BAI09B03), Beijing Municipal Administration of Hospitals' Mission Plan (SML20150501), Beijing Institute for Brain Disorders (BIBDPXM2013_014226_07_000084), and the Program of the National Natural Science Foundation of China (81371292).

\section{References}

1. Adeoye O, Knight WA, Khoury J, Schmit PA, Sucharew H, Broderick JP, et al: A matched comparison of eptifibatide plus rt-PA versus rt-PA alone in acute ischemic stroke. J Stroke Cerebrovasc Dis 23:e313-e315, 2014

2. Amlie-Lefond C, Ellenbogen RG: Factors associated with the presentation of moyamoya in childhood. J Stroke Cerebrovasc Dis 24:1204-1210, 2015

3. Arias EJ, Derdeyn CP, Dacey RG Jr, Zipfel GJ: Advances and surgical considerations in the treatment of moyamoya disease. Neurosurgery 74 (Suppl 1):S116-S125, 2014

4. Arias EJ, Dunn GP, Washington CW, Derdeyn CP, Chicoine MR, Grubb RL Jr, et al: Surgical revascularization in North American adults with moyamoya phenomenon: long-term angiographic follow-up. J Stroke Cerebrovasc Dis 24:15971608,2015

5. Chiu D, Shedden P, Bratina P, Grotta JC: Clinical features of moyamoya disease in the United States. Stroke 29:13471351,1998

6. Cho WS, Kim JE, Kim CH, Ban SP, Kang HS, Son YJ, et al: Long-term outcomes after combined revascularization surgery in adult moyamoya disease. Stroke 45:3025-3031, 2014

7. Dusick JR, Gonzalez NR, Martin NA: Clinical and angiographic outcomes from indirect revascularization surgery for Moyamoya disease in adults and children: a review of 63 procedures. Neurosurgery 68:34-43, 2011

8. Fujimura M, Mugikura S, Kaneta T, Shimizu H, Tominaga T: Incidence and risk factors for symptomatic cerebral hyperperfusion after superficial temporal artery-middle cerebral artery anastomosis in patients with moyamoya disease. Surg Neurol 71:442-447, 2009

9. Gross BA, Du R: Adult moyamoya after revascularization. Acta Neurochir (Wien) 155:247-254, 2013

10. Han DH, Kwon OK, Byun BJ, Choi BY, Choi CW, Choi JU, et al: A co-operative study: clinical characteristics of 334 Korean patients with moyamoya disease treated at neurosurgical institutes (1976-1994). Acta Neurochir (Wien) 142:1263-1274, 2000

11. Hashimoto N, Tominaga T, Miyamoto S, Nagata I, Houkin K, Suzuki N, et al: Guidelines for diagnosis and treatment of moyamoya disease (spontaneous occlusion of the circle of Willis). Neurol Med Chir (Tokyo) 52:245-266, 2012

12. Huang Z, Ding X, Men W, Zhang D, Zhao Y, Wang R, et al: Clinical features and outcomes in 154 patients with haemorrhagic moyamoya disease: comparison of conservative treatment and surgical revascularization. Neurol Res 37:886892,2015

13. Imai H, Miyawaki S, Ono H, Nakatomi H, Yoshimoto Y, Saito N: The importance of encephalo-myo-synangiosis in surgical revascularization strategies for moyamoya disease in children and adults. World Neurosurg 83:691-699, 2015

14. Jo KI, Yeon JY, Hong SC, Kim JS: Clinical course of asymptomatic adult moyamoya disease. Cerebrovasc Dis 37:94101,2014

15. Khan N, Achrol AS, Guzman R, Burns TC, Dodd R, BellStephens T, et al: Sex differences in clinical presentation and treatment outcomes in Moyamoya disease. Neurosurgery 71:587-593, 2012

16. Kim DS, Kang SG, Yoo DS, Huh PW, Cho KS, Park CK: Surgical results in pediatric moyamoya disease: angiographic revascularization and the clinical results. Clin Neurol Neurosurg 109:125-131, 2007

17. Kobayashi E, Saeki N, Oishi H, Hirai S, Yamaura A: Longterm natural history of hemorrhagic moyamoya disease in 42 patients. J Neurosurg 93:976-980, 2000

18. Kuriyama S, Kusaka Y, Fujimura M, Wakai K, Tamakoshi A, Hashimoto S, et al: Prevalence and clinicoepidemiological features of moyamoya disease in Japan: findings from a nationwide epidemiological survey. Stroke 39:42-47, 2008

19. Kuroda S, Houkin K: Moyamoya disease: current concepts and future perspectives. Lancet Neurol 7:1056-1066, 2008

20. Lee SB, Kim DS, Huh PW, Yoo DS, Lee TG, Cho KS: Longterm follow-up results in 142 adult patients with moyamoya disease according to management modality. Acta Neurochir (Wien) 154:1179-1187, 2012

21. Liu X, Zhang D, Shuo W, Zhao Y, Wang R, Zhao J: Long term outcome after conservative and surgical treatment of haemorrhagic moyamoya disease. J Neurol Neurosurg Psychiatry 84:258-265, 2013

22. Macyszyn L, Attiah M, Ma TS, Ali Z, Faught R, Hossain $A$, et al: Direct versus indirect revascularization procedures for moyamoya disease: a comparative effectiveness study. J Neurosurg [epub ahead of print July 29, 2016. DOI: 10.3171/2015.8.JNS15504]

23. Matsushima T, Inoue K, Kawashima M, Inoue T: History of the development of surgical treatments for moyamoya disease. Neurol Med Chir (Tokyo) 52:278-286, 2012

24. Noah MA, Peek GJ, Finney SJ, Griffiths MJ, Harrison DA, Grieve R, et al: Referral to an extracorporeal membrane oxygenation center and mortality among patients with severe 2009 influenza A(H1N1). JAMA 306:1659-1668, 2011

25. Noh HJ, Kim SJ, Kim JS, Hong SC, Kim KH, Jun P, et al: Long term outcome and predictors of ischemic stroke recurrence in adult moyamoya disease. J Neurol Sci 359:381-388, 2015

26. Normand ST, Landrum MB, Guadagnoli E, Ayanian JZ, Ryan TJ, Cleary PD, et al: Validating recommendations for coronary angiography following acute myocardial infarction in the elderly: a matched analysis using propensity scores. $\mathbf{J}$ Clin Epidemiol 54:387-398, 2001

27. Park EK, Lee YH, Shim KW, Choi JU, Kim DS: Natural history and progression factors of unilateral moyamoya disease in pediatric patients. Childs Nerv Syst 27:1281-1287, 2011

28. Qian C, Yu X, Li J, Chen J, Wang L, Chen G: The efficacy of surgical treatment for the secondary prevention of stroke in symptomatic moyamoya disease: a meta-analysis. Medicine (Baltimore) 94:e2218, 2015

29. Scott RM, Smith ER: Moyamoya disease and moyamoya syndrome. N Engl J Med 360:1226-1237, 2009

30. Sposito C, Battiston C, Facciorusso A, Mazzola M, Muscarà C, Scotti M, et al: Propensity score analysis of outcomes following laparoscopic or open liver resection for hepatocellular carcinoma. Br J Surg 103:871-880, 2016

31. Sundaram S, Sylaja PN, Menon G, Sudhir J, Jayadevan ER, Sukumaran S, et al: Moyamoya disease: a comparison of long term outcome of conservative and surgical treatment in India. J Neurol Sci 336:99-102, 2014

32. Suzuki J, Takaku A: Cerebrovascular "moyamoya" disease. 
Disease showing abnormal net-like vessels in base of brain. Arch Neurol 20:288-299, 1969

33. Teo MK, Madhugiri VS, Steinberg GK: Editorial. Direct versus indirect bypass for moyamoya disease: ongoing controversy. J Neurosurg [epub ahead of print July 29, 2016. DOI: 10.3171/2015.8.JNS15504]

34. Uchino H, Kim JH, Fujima N, Kazumata K, Ito M, Nakayama N, et al: Synergistic interactions between direct and indirect bypasses in combined procedures: the significance of indirect bypasses in moyamoya disease. Neurosurgery 80:201-209, 2017

\section{Disclosures}

The authors report no conflict of interest concerning the materials or methods used in this study or the findings specified in this paper.

\section{Author Contributions}

Conception and design: J Zhao, Deng, Gao, D Zhang, Y Zhang, R Wang, S Wang, Cao, Y Zhao, Q Zhang. Acquisition of data: J Zhao, Deng, Gao, Liu, Q Zhang. Analysis and interpretation of data: J Zhao, Deng, Gao, D Zhang, Y Zhang, R Wang, Y Zhao, Liu. Drafting the article: Deng, Gao. Critically revising the article: J Zhao, Deng, Gao, D Zhang, Y Zhang, R Wang, S Wang, Cao, Y Zhao, Pan, Q Zhang. Reviewed submitted version of manuscript: J Zhao, Deng, Gao. Approved the final version of the manuscript on behalf of all authors: J Zhao. Statistical analysis: Deng, Gao, Pan. Administrative/technical/material support: J Zhao, Deng, Gao. Study supervision: J Zhao, Deng, Gao.

\section{Correspondence}

Jizong Zhao, Department of Neurosurgery, Beijing Tiantan Hospital, Capital Medical University, No. 6 Tiantan Xili, Dongcheng District, Beijing 100050, China. email: zhaojz205@163.com. 